\title{
SINGULARLY PERTURBED PERIODIC PARABOLIC EQUATIONS WITH ALTERNATING BOUNDARY LAYER TYPE SOLUTIONS
}

\author{
ADELAIDA B. VASIL'EVA AND LEONID V. KALACHEV
}

Received 30 September 2004; Accepted 4 November 2004

We consider a class of singularly perturbed parabolic equations for which the degenerate equations obtained by setting the small parameter equal to zero are algebraic equations that have several roots. We study boundary layer type solutions that, as time increases, periodically go through two fairly long lasting stages with extremely fast transitions in between. During one of these stages the solution outside the boundary layer is close to one of the roots of the degenerate (reduced) equation, while during the other stage the solution is close to the other root. Such equations may be used as models for bio-switches where the transitions between various stationary states of biological systems are initiated by comparatively slow changes within the systems.

Copyright (c) 2006 A. B. Vasil'eva and L. V. Kalachev. This is an open access article distributed under the Creative Commons Attribution License, which permits unrestricted use, distribution, and reproduction in any medium, provided the original work is properly cited.

\section{Introduction}

Parabolic equations with several possible locally stable stationary solutions are often used for modeling of biological switches (i.e., biological systems where transitions may occur between various biologically meaningful stationary states); see, for example, Okubo and Levin [5], Keener and Sneyd [2], Murray [3]. A particular current state of a bio-switch is determined by the original initial condition that belongs to a domain of attraction of this stable stationary state. Usually, to initiate a transition to another stationary state of the biological system a perturbation of a dependent variable is required that translates the instantaneous solution to the domain of attraction of this new stable stationary state.

Here we present a mathematical model that would describe a different type of transition between various states of the bio-switches. Unlike the cases mentioned above, these new transitions are initiated by comparatively slow changes that occur within biological systems (and caused, e.g., by the seasonal temperature, humidity, vegetation, etc.). In the present paper we concentrate on a heuristic analysis of this new phenomena. Some short 
discussion on sensitivity and tuning of bio-switches is given at the end of the Conclusions Section.

Specifically, in this paper we present formal results on the qualitative asymptotic analysis of alternating boundary layer type solutions of the bistable scalar parabolic equations with periodic dependence. Our main goals are (1) to show how such solutions appear in the cases where the non-linearity in the equation does not contain explicit dependence on spatial variable, $x$, and (2) to clarify the effect of the presence of a slow convectionlike term on the initiation of fast transition between the two longer lasting states of the periodic solution. During these stages the solution outside the boundary layer is close to different roots of the nonlinearity. The main techniques of study used here are the boundary function method algorithm (see Vasil'eva et al. [7]) and the phase-plane analysis of the time dependent orbits describing the boundary layers near the endpoints of the spatial interval of interest (here, $x \in[0,1]$ ).

When the right-hand side of the parabolic equation does not depend explicitly on the spatial variable, $x$, the moving transition thresholds start symmetrically on both sides of the spatial interval. This is not the case when the small convection-like term is present in the equation. Depending on the sign of the convection-like term, the transition will now start either on the left or on the right boundary of the spatial interval.

In this paper we only briefly discuss the structure of the fast moving transition layer that accompanies the switching between the two mentioned above longer lasting stages of the periodic solution. A more detailed description of the periodic traveling waves for reaction-diffusion equations can be found, for example, in Alikakos et al. [1].

The paper is organized as follows. A brief overview of some recent results on boundary layer type solutions of singularly perturbed parabolic equations is given in Section 2 . The new results on alternating boundary layer type solutions are presented in Section 3. Conclusions following from the analysis are discussed in Section 4.

\section{Boundary layer type solutions of singularly perturbed parabolic equations}

2.1. Equation without explicit dependence on $u_{x}$ (the case where degenerate equation has one root). Let us consider a problem for parabolic equation with Dirichlet type boundary conditions and periodic conditions in $t(0<\varepsilon \ll 1$ is a small parameter $)$ :

$$
\begin{gathered}
\varepsilon^{2}\left(u_{x x}-u_{t}\right)=F(u, x, t), \quad 0<x<1, \\
u(0, t, \varepsilon)=u(1, t, \varepsilon)=0, \\
u(x, t, \varepsilon)=u(x, t+2 \pi, \varepsilon)=0 .
\end{gathered}
$$

Let the following assumptions hold.

(A1) Function $F(u, x, t)$ is periodic in $t$ and is continuous together with its first derivatives in domain

$$
G=\left\{(x, t) \in D=[0,1] \times R_{1},|u| \leq K\right\}
$$

(A2) The degenerate equation $F(u, x, t)=0$ has a root $\phi(x, t)$ in domain $D$, such that $F_{u}(\phi(x, t), x, t)>0$. 
Next, consider the sum

$$
\begin{aligned}
U(x, t, \varepsilon) & =\phi(x, t)+\Pi\left(\eta_{0}, t\right)+R\left(\eta_{1}, t\right) \\
& =\phi(x, t)+\Pi\left(\frac{x}{\varepsilon}, t\right)+R\left(\frac{1-x}{\varepsilon}, t\right) .
\end{aligned}
$$

Here $\eta_{0}=x / \varepsilon, \eta_{1}=(1-x) / \varepsilon$ are the, so-called, stretched variables, and $\Pi, R$ are the boundary functions. The left boundary function, $\Pi$, is determined as a solution of the following problem:

$$
\begin{aligned}
\frac{d^{2} \Pi}{d \eta_{0}^{2}} & =F(\phi(0, t)+\Pi, 0, t), \\
\Pi(0, t) & =-\phi(0, t),\left.\quad \Pi\right|_{\eta_{0} \rightarrow \infty} \longrightarrow 0 .
\end{aligned}
$$

In this problem $t$ is a parameter.

Consider a phase plane (,$\left.d \Pi / d \eta_{0}\right)$ for (2.6) ( $t$ fixed). By virtue of assumption (A2) the point $(0,0)$ (an equilibrium point for $(2.6))$ is a saddle.

(A3) Let the vertical line $\Pi=-\phi(0, t)$ intersect the separatrix entering the saddle point $(0,0)$ as $\eta_{0} \rightarrow \infty$.

The right boundary function, $R$, is found as a solution of the problem

$$
\begin{aligned}
\frac{d^{2} R}{d \eta_{1}^{2}} & =F(\phi(1, t)+R, 1, t), \\
R(0, t) & =-\phi(1, t),\left.\quad R\right|_{\eta_{1} \rightarrow \infty} \longrightarrow 0 .
\end{aligned}
$$

In the phase plane $\left(R, d R / d \eta_{1}\right)$ of the above equation the point $(0,0)$ is also a saddle.

(A4) Let the vertical line $R=-\phi(1, t)$ intersect the separatrix entering the saddle point $(0,0)$ as $\eta_{1} \rightarrow \infty$.

The following theorem holds (see, e.g., Nefedov [4]).

THEOREM 2.1. Under conditions (A1)-(A4) and for sufficiently small $\varepsilon$ there exists a boundary layer type periodic solution $u(x, t, \varepsilon)$ of the problem (2.1)-(2.3) such that

$$
|u(x, t, \varepsilon)-U(x, t, \varepsilon)|<C \varepsilon, \quad \text { for }(x, t) \in D,
$$

where $C>0$ is a constant that does not depend on $\varepsilon$.

Here $U(x, t, \varepsilon)$ is defined by $(2.5)$.

2.2. Equation with weak dependence on $u_{x}$ (the case where the $\varepsilon=0$ equation has one root). Now we consider the equation

$$
\varepsilon^{2}\left(u_{x x}-u_{t}\right)=F\left(\varepsilon u_{x}, u, x, t\right), \quad 0<x<1
$$

with the same additional conditions (2.2), (2.3). Equation (2.9) differs from (2.1) by an extra argument $\varepsilon u_{x}$ in the right-hand side. 
The new assumptions $\left(\mathrm{A} 1^{*}\right)$ and $\left(\mathrm{A} 2^{*}\right)$ must be formulated for $(2.9)$, and they change only slightly compared to corresponding assumptions (A1) and (A2) for (2.1). In particular, $\left(\mathrm{A} 1^{*}\right)$ differs from (A1) by specification of a new domain on which function $F(z, u, x, t)$ must be periodic in $t$ and be continuous together with its first derivatives:

$$
G=\left\{(x, t) \in D=[0,1] \times R_{1},|u| \leq K,|z|<K\right\} .
$$

In the new assumption (A2*), compared to (A2), function $F(z, u, x, t)$ will have an additional argument $z$ that is set to zero: $F(0, u, x, t)=0$ has a root $\phi(x, t)$, such that

$$
F_{u}(0, \phi(x, t), x, t)>0 \text {. }
$$

Instead of problem (2.6), we now consider

$$
\begin{aligned}
\frac{d^{2} \Pi}{d \eta_{0}^{2}} & =F\left(\frac{d \Pi}{d \eta_{0}}, \phi(0, t)+\Pi, 0, t\right), \\
\Pi(0, t) & =-\phi(0, t),\left.\quad \Pi\right|_{\eta_{0} \rightarrow \infty} \longrightarrow 0,
\end{aligned}
$$

and instead of problem (2.7), we consider

$$
\begin{aligned}
\frac{d^{2} R}{d \eta_{1}^{2}} & =F\left(\frac{d R}{d \eta_{1}}, \phi(1, t)+R, 1, t\right), \\
R(0, t) & =-\phi(1, t),\left.\quad R\right|_{\eta_{1} \rightarrow \infty} \longrightarrow 0 .
\end{aligned}
$$

The assumptions (A3), (A4) will stay the same as in the previous subsection.

The difference between (2.12), (2.13) and (2.6), (2.7) is in that the problems (2.6) and (2.7) have the first integral that can be found explicitly, while problems (2.12) and (2.13) do not possess this property. In what follows, this difference will play an important role.

The following theorem holds (see Vasil'eva [6]).

THEOREM 2.2. Under conditions $\left(A 1^{*}\right),\left(A 2^{*}\right),(A 3),(A 4)$ and for sufficiently small $\varepsilon$ there exists a boundary layer type periodic solution $u(x, t, \varepsilon)$ of the problem (2.9), (2.2), (2.3) which is asymptotically close to $\phi(x, t)$ for $0<x<1$.

We note that this theorem is weaker than Theorem 2.1 since it does not provide us with asymptotics of the solution (i.e., the rate of convergence is not indicated).

2.3. The bistable case. Let the equation $F(u, x, t)=0$ have several roots. We consider in more detail the case of three roots in $D$ :

$$
\begin{aligned}
& \phi_{1}(x, t)<\phi_{2}(x, t)<\phi_{3}(x, t), \\
& \left.\frac{\partial F}{\partial u}\right|_{u=\phi_{i}}>0 \quad(i=1,3),\left.\quad \frac{\partial F}{\partial u}\right|_{u=\phi_{2}}<0 .
\end{aligned}
$$

Now the problem for parabolic equation (2.1) may have a solution described in Section 2.1 with $\phi=\phi_{1}(x, t)$, as well as a solution with $\phi=\phi_{3}(x, t)$. These two solutions may co-exist.

Similar phenomena are also observed in the case where $F=F\left(\varepsilon u_{x}, u, x, t\right)$ and the $\varepsilon=0$ equation $F(0, u, x, t)=0$ has three roots (see Vasil'eva [6]). 


\section{Break down of the boundary layer type solution}

3.1. Bistable I. Consider the bistable equation

$$
\varepsilon^{2}\left(u_{x x}-u_{t}\right)=\left(u^{2}-1\right)(u-\phi(t)), \quad 0<x<1,
$$

with conditions (2.2), (2.3). Here $\phi_{1}=-1, \phi_{2}=\phi(t)$, and $\phi_{3}=1$. We assume that $-1<$ $\phi(t)<1$ is a periodic function of $t$, and that conditions (A1)-(A4) hold. Since the form of the right-hand side in (3.1) is now specified, we may explain the meaning of conditions (A3), (A4) in more detail.

Consider, for example, requirement (A3). Let us construct separatrices on the phase plane for corresponding equation mentioned in (A3). For convenience, we introduce a new variable, $\tilde{u}=\phi_{3}+\Pi$. (Here without loss of generality we start with the discussion of the boundary layer type solution that is close to $\phi_{3}$ outside the boundary layer. We could have chosen to start our discussion with the solution that is close to $\phi_{1}$ as well. Note that in Section 2.1, in problem (2.6), only the case of a unique root of the degenerate equation was mentioned.) Now the problem (2.6) will have the form

$$
\begin{gathered}
\frac{d^{2} \tilde{u}}{d \eta_{0}^{2}}=\left(\tilde{u}^{2}-1\right)(\tilde{u}-\phi(t)), \\
\tilde{u}(0, t)=0,\left.\quad \tilde{u}\right|_{\eta_{0} \rightarrow \infty} \longrightarrow 1 \quad(t \text { is a parameter }) .
\end{gathered}
$$

The first integral of this equation can be written out explicitly:

$$
\frac{p^{2}}{2}=\int_{1}^{\tilde{u}}\left(s^{2}-1\right)(s-\phi(t)) d s
$$

(here $\left.p=d \tilde{u} / d \eta_{0}\right)$.

Expression (3.3) describes separatrices passing through the saddle $\tilde{u}=1, p=0$. The changes in the phase portrait of the equation that occur with changing $t$ are shown in Figure 3.1. (To make notation shorter in this figure as well as in the figures below tilde is omitted, that is, in the figures $u$ is used instead of $\tilde{u}$.) The arrows show the direction corresponding to increasing values of $\eta_{0}$. The value $\tilde{u}=\psi$ is an abscissa of a point corresponding to intersection of a homoclinic orbit loop with the $\tilde{u}$-axis $(p=0)$ and lying to the left of $\tilde{u}=1$.

Let us start with the case where $\phi(t)$ increases with $t$. In Figure 3.1(a) condition (A3) is satisfied: the vertical line $\tilde{u}=0$ intersects the separatrix $L$ entering the saddle $(1,0)$ for $\eta_{0} \rightarrow \infty$.

As $\phi(t)$ continues to increase, we arrive at the critical case, shown in Figure 3.1(b), where the vertical line $\tilde{u}=0$ only touches the separatrix $L$ (at the turning point of the homoclinic loop).

In Figure 3.1(c) the value of $\phi(t)$ is so large that the vertical line $\tilde{u}=0$ does not intersect the separatrix $L$ any more.

Thus, condition (A3) holds for cases shown in Figures 3.1(a) and (b), and Figure 3.1(b) corresponds to the maximal value $t=t_{1}$ for which (A3) is still true. For $t=t_{1}+0$ the solution with left boundary layer transition connecting the boundary condition $\left.\tilde{u}\right|_{x=0}=0$ with $\phi_{3}=1$ breaks down, and a new boundary layer transition connecting $\left.\tilde{u}\right|_{x=0}=0$ with 


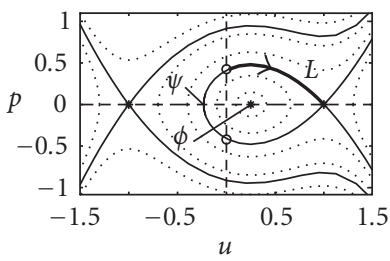

(a)

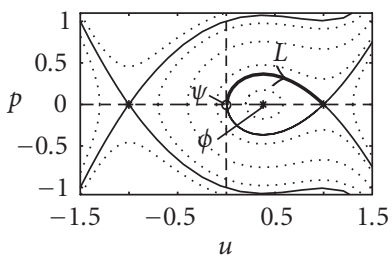

(b)

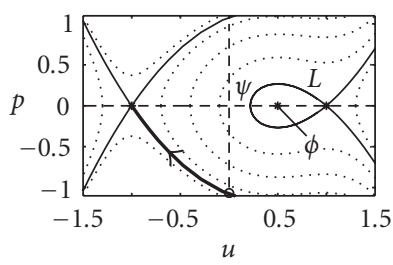

(c)

Figure 3.1. Bold solid lines represent phase trajectories corresponding to the boundary layer transitions near the left boundary point $x=0$.

$\phi_{1}=-1$ appears (a separatrix entering the saddle $(-1,0)$ and intersecting $\left.\tilde{u}\right|_{x=0}=0$ exists for some $t<t_{1}$ as well, however, it does not correspond to a boundary layer transition for $\left.t<t_{1}\right)$. This new left boundary layer transition is defined as a solution of (3.2) with $\tilde{u}=\phi_{1}+\Pi$ and the new condition $\tilde{u} \rightarrow-1$ as $\eta_{0} \rightarrow \infty$.

Near the boundary point $x=1$ a similar picture is observed (the behavior analogous to that presented in Figures 3.1(a), (b), (c), for the separatrix entering the saddle for $\eta_{1} \rightarrow$ $\infty$, may be analyzed as well). So, the upper boundary layer type solution breaks down simultaneously at both endpoints, $x=0$ and $x=1$, of the spatial domain of interest (this happens due to the fact that $\phi_{2}=\phi(t)$ is independent of $x$ ).

The formula for the instant of time $t=t_{1}$ when the break down occurs can be derived analytically. From (3.3), we obtain

$$
\begin{aligned}
\frac{p^{2}}{2} & =\frac{1}{4}\left(\tilde{u}^{4}-1\right)-\frac{\phi}{3}\left(\tilde{u}^{3}-1\right)-\frac{1}{2}\left(\tilde{u}^{2}-1\right)+\phi(\tilde{u}-1) \\
& =(\tilde{u}-1)^{2}\left[\frac{1}{4}(\tilde{u}+1)^{2}-\frac{\phi}{3}(\tilde{u}+2)\right] .
\end{aligned}
$$

The value of $\psi$ is obtained from the above expression as follows: for $p=0$ the term in the square brackets must be zero. Thus,

$$
\left[\frac{1}{4}(\tilde{u}+1)^{2}-\frac{\phi}{3}(\tilde{u}+2)\right]=0
$$


and then,

$$
\tilde{u}=\psi=-1+\frac{2}{3}\left(\phi \pm \sqrt{\phi^{2}+3 \phi}\right) .
$$

In the critical situation (shown in Figure 3.1(b)), $\psi=0$, which leads to $\phi=3 / 8$ (the second root is omitted since it is negative, and it lies to the left of $\tilde{u}=-1)$. Now, the particular value of $t_{1}$ for which the upper boundary layer type solution (i.e., the one that tends to root $\phi_{3}=1$ in the interior of spatial domain of interest as $\varepsilon \rightarrow 0$ ) breaks down is found from the equation

$$
\phi\left(t_{1}\right)=\frac{3}{8}
$$

For $t=t_{1}+0$ the connection of a point corresponding to the boundary condition at $x=$ 0 (where $\tilde{u}=0$ ) with the saddle point $\left(\phi_{3}, 0\right)=(1,0)$ via one separatrix breaks down, but the connection via another separatrix with the saddle $\left(\phi_{1}, 0\right)=(-1,0)$ remains (see Figures 3.1(b) and (c)), and the lower boundary layer type solution starts to develop. This phenomenon is illustrated in Figure 3.2 where the instantaneous profiles of the solution are shown in coordinates $(x, u)$ for certain values of $t$.

We may note that this phenomenon is symmetric with respect to boundaries $x=0$ and $x=1$. In Figure 3.2(a) we show the upper boundary layer type solution; in Figure 3.2(b) the critical form of the upper boundary layer type solution is presented (the solution in this case has zero derivative, $p(\psi)=0$, at the boundaries); in Figure 3.2(c) we illustrate the appearance of lower boundary layer type solution via moving thresholds; and finally, in Figure 3.2(d) the developed form of lower boundary layer type solution is shown. The function $\phi(t)$ is periodic. So, after reaching some maximal value $3 / 8<\phi_{\max }<1$ it will start to decrease. Here we address the case where for the minimal value of $\phi(t)$ the following condition holds: $-1<\phi_{\min }<-3 / 8$. Then, after stage $(\mathrm{d})$ the process similar to that described in Figures 3.2(a)-(d) and corresponding to a transition from lower to upper boundary layer type solution is observed. This latter transition completes the full cycle.

Thus, for (3.1) with conditions (2.2), (2.3) we have the situation for which the boundary layer type solution of alternating type exits: for this problem the upper boundary layer type solution at a certain instant of time is being transformed to the lower boundary layer type solution which, in turn, at another instant of time transforms back into the upper boundary layer type solution. This process repeats itself periodically in time. The full analytical description of the process shown in Figures 3.2(a)-(d) is still open.

In the paper by Vasil'eva et al. [8] a method is given that describes the solution of the moving layer type appearing in stage (b) and further developing in stage (c) (see Figures 3.2(b), (c)). Following [8], consider the expression

$$
\bar{u}=\frac{1-\exp \left[(-\sqrt{2} / \varepsilon)\left(x-r_{0}\right)\right]}{1+\exp \left[(-\sqrt{2} / \varepsilon)\left(x-r_{0}\right)\right]} .
$$

If $r_{0}$ is considered to be a parameter, then $\bar{u}$ is a solution of the equation

$$
\frac{d^{2} \bar{u}}{d \eta^{2}}=\left(\bar{u}^{2}-1\right) \bar{u},
$$




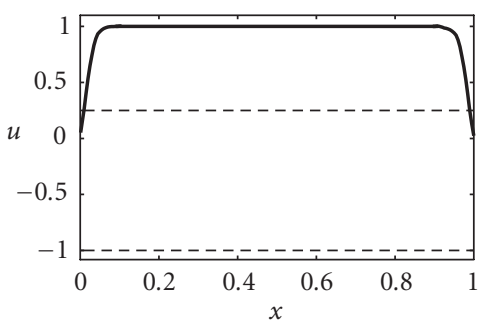

(a)

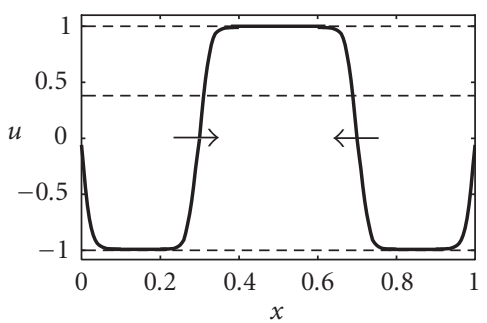

(c)

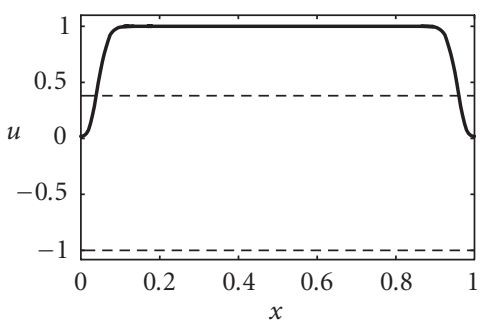

(b)

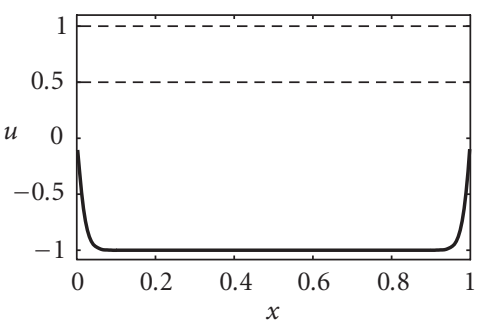

(d)

Figure 3.2. Sample phases of the periodic behavior illustrating the switching between upper and lower boundary layer type solutions.

that has the form of a transition layer since

$$
\lim _{\varepsilon \rightarrow 0} \bar{u} \longrightarrow \begin{cases}-1, & x<r_{0}, \\ 1, & x>r_{0} .\end{cases}
$$

It turns out that if $r_{0}=r_{0}(t, \varepsilon)$ is defined by the equation

$$
\varepsilon \frac{d r_{0}}{d t}=\sqrt{2} \phi(t)
$$

the expression (3.8), where $r_{0}=r_{0}(t, \varepsilon)$ from (3.11) is substituted for $r_{0}$, satisfies (3.1) with discrepancy of order $O(\varepsilon)$. This solution could be constructed so as to satisfy the initial condition $r_{0}\left(t_{1}, \varepsilon\right)=0$. Let $\phi(t)$ change with period $2 \pi$, and for $t=t_{1}>0$ equal $3 / 8$. For some $t$ it attains its maximal value $\phi_{\max }<1$, then starts to decrease, passes through zero in the direction of negative values of $\phi(t)$ until it reaches its minimal value $-1<\phi_{\min }<0$ (this minimal value could, e.g., be $\phi_{\min }=-\phi_{\max }$ ). After that $\phi(t)$ starts to increase and becomes $3 / 8$ again for $t=t_{1}+2 \pi$. The solution of (3.11) has the form

$$
r_{0}(t, \varepsilon)=\frac{\sqrt{2}}{\varepsilon} \int_{t_{1}}^{t} \phi(\theta) d \theta
$$


Function $r_{0}(t, \varepsilon)$ has a singularity of order $1 / \varepsilon$, that is, the motion of the layer occurs at a very fast time scale. For $t=t_{1}$ and $x=0$, we have $\bar{u}=0$, so we obtain stage (b) of the time evolution of the solution that is followed by stage (c).

The layer connecting 1 on the left to 0 on the right is given by

$$
\begin{gathered}
\hat{u}=\frac{1-\exp \left[(\sqrt{2} / \varepsilon)\left(x-r_{1}\right)\right]}{1+\exp \left[(\sqrt{2} / \varepsilon)\left(x-r_{1}\right)\right]} \\
\varepsilon \frac{d r_{1}}{d t}=-\sqrt{2} \phi(t), \quad r_{1}(t, \varepsilon)=-\frac{\sqrt{2}}{\varepsilon} \int_{t_{1}}^{t} \phi(\theta) d \theta+1 .
\end{gathered}
$$

Similar to the case of $r_{0}$, for $t=t_{1}+0$, that is, just after $\phi(t)>0$ reaches $3 / 8$, the value of $r_{1}$ starts to decay rapidly away from 1 .

Mutual destruction of the two moving layers takes place at the end of stage (c) (see Figure 3.2(c)). This transition to a lower boundary layer type solution occurs for $r=1 / 2$ (due to symmetry), and at an instant of time $t_{2}$ defined by the equation

$$
\frac{\varepsilon}{2}=\sqrt{2} \int_{t_{1}}^{t_{2}} \phi(\theta) d \theta
$$

that is, $t_{2}-t_{1}=O(\varepsilon)$.

After that, the stage corresponding to the lower boundary layer type solution starts (Figure 3.4 (d)). This stage lasts until it breaks down at an instant of time defined by the equation (for $t_{3}$ ): $\phi\left(t_{3}\right)=-3 / 8$. This equation is derived similar to (3.7). At an instant of time $t_{3}$ the transition back to the upper boundary layer type solution is initiated (this transition process goes through the steps similar to those shown in Figure 3.2). Thus, the duration of the transition stage (from one boundary layer type solution to the other) is very short ( of order $O(\varepsilon)$ ), while the duration of stages corresponding to upper and lower boundary layer type solutions is equal approximately to $\pi$. In the literature (see Vasil'eva et al. [8]) the fast transition stage is usually referred to as a run, and the slow longer lasting boundary layer type stage is called a halt. We note that in [8] a somewhat different case is considered, but the terminology introduced there may be applied here as well.

In Figure 3.3 we show schematic representations of phase portraits for the boundary layer transitions corresponding to the stages of time evolution of the periodic solution discussed above. The bold solid lines correspond to the left boundary layer transitions (near $x=0$ ), and the dashed lines correspond to the right boundary layer transitions (near $x=1$ ). Arrows show the directions of increase in $\eta_{0}$ (solid line) and in $\eta_{1}$ (dashed line).

The following values of $\phi(t)$ produce various phase portraits shown in Figure 3.3:

(a) $\phi(t)=3 / 8$ - maximal critical value; the start of a run; switching from upper to lower boundary layer type solution;

(b) $3 / 8<\phi(t)<\phi_{\max }$-increase of $\phi(t)$;

(c) $0<\phi(t)<\phi_{\max }$-decrease of $\phi(t)$;

(d) $\phi(t)=0$-value of $\phi(t)$ for which heteroclinic connections exist;

(e) $-3 / 8<\phi(t)<0$-value of $\phi(t)$ continues to decrease;

(f) $\phi(t)=-3 / 8$ - minimal critical value; the start of a run; switching from lower to upper boundary layer type solution; 


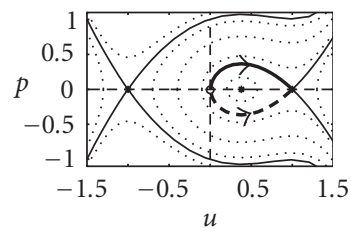

(a)

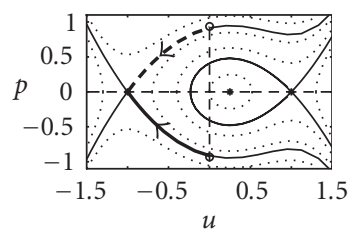

(c)

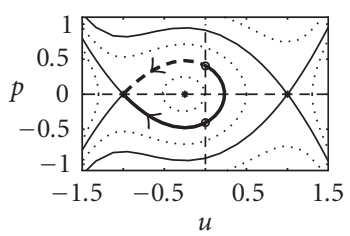

(e)

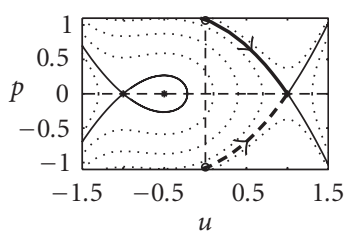

(g)

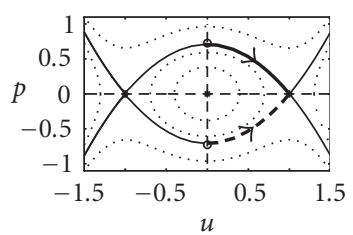

(i)

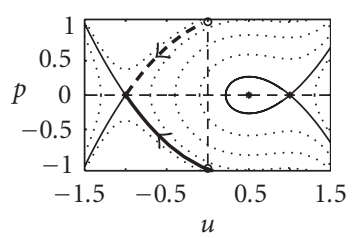

(b)

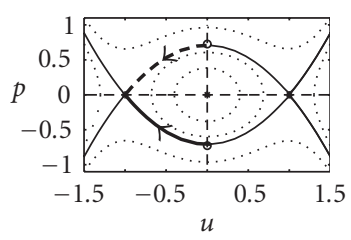

(d)

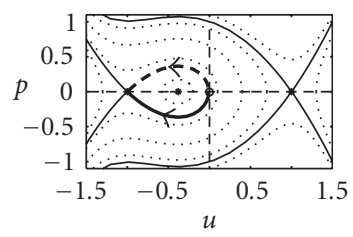

(f)

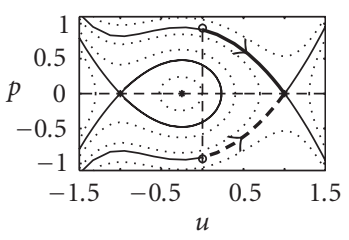

(h)

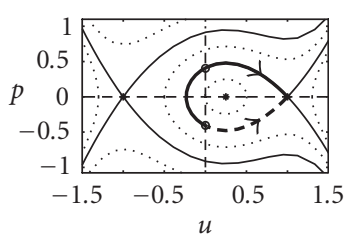

(j)

Figure 3.3. Phase portraits illustrating time dynamics of the boundary layer transitions present near the left (solid line) and the right (dashed line) boundaries of the interval $x \in[0,1]$. 
(g) $\phi_{\min }<\phi(t)<-3 / 8$ - decrease of $\phi(t)$;

(h) $\phi_{\min }<\phi(t)<0$-increase of $\phi(t)$;

(i) $\phi(t)=0$-value of $\phi(t)$ for which heteroclinic connections exist;

(j) $0<\phi(t)<3 / 8$-increase of $\phi(t)$.

Stage (a) follows stage (j), which completes the cycle. We note that the phase portraits (g), (h), (i), (j) shown in Figure 3.3 correspond to various stages of solution phase (a) presented in Figure 3.2, the phase portrait (a) in Figure 3.3 corresponds to solution phase (b) in Figure 3.2, the phase portraits (b), (c), (d), (e) in Figure 3.3 correspond to various stages of solution phase (d) in Figure 3.2.

3.2. Bistable II. Next we consider the equation

$$
\varepsilon^{2}\left(u_{x x}-u_{t}\right)=\varepsilon a u_{x}+\left(u^{2}-1\right)(u-\phi(t)), \quad 0<x<1,
$$

with the same conditions (2.2), (2.3). The difference with (3.1) is due to the convection term $\varepsilon a u_{x}$. Here we begin with taking $a=$ const $>0$.

For (3.15) the formula of type (3.3) does not exist since, unlike (3.2), equation

$$
\frac{d^{2} \tilde{u}}{d \eta_{0}^{2}}=a \frac{d \tilde{u}}{d \eta_{0}}+\left(\tilde{u}^{2}-1\right)(\tilde{u}-\phi(t)),
$$

with conditions

$$
\tilde{u}(0, t)=0,\left.\quad \tilde{u}\right|_{\eta_{0} \rightarrow \infty} \longrightarrow 1(t \text { is a parameter }),
$$

cannot be integrated in quadratures. Thus, the value for $\phi\left(t_{1}\right)$ cannot be found explicitly (cf. (3.7)).

Simple phase plane analysis can be performed for (3.16). Corresponding steady states of the equivalent equation

$$
p \frac{d p}{d \tilde{u}}=a p+\left(\tilde{u}^{2}-1\right)(\tilde{u}-\phi)
$$

on the $(\tilde{u}, p)$-plane are found analogously to the case of $(3.1)$. They are $(-1,0),(1,0)$, and $(\phi, 0)$. Once again, $(-1,0)$ and $(1,0)$ are saddles. However, $(\phi, 0)$ now is either a focus (for not too large values of $a$ ) or a node.

Eigenvalues of the Jacobian matrix in the case of $(\phi, 0)$ are defined by the equation

$$
\operatorname{det}\left(\begin{array}{cc}
-\lambda & 1 \\
\left(\phi^{2}-1\right) & a-\lambda
\end{array}\right) \equiv \lambda^{2}-a \lambda-\left(\phi^{2}-1\right)=0
$$

that is,

$$
\lambda_{1,2}=\frac{a}{2} \pm \sqrt{\frac{a^{2}}{4}+\left(\phi^{2}-1\right)}
$$

and under condition $a^{2}<4\left(1-\phi^{2}\right)$ we obtain an unstable focus. 


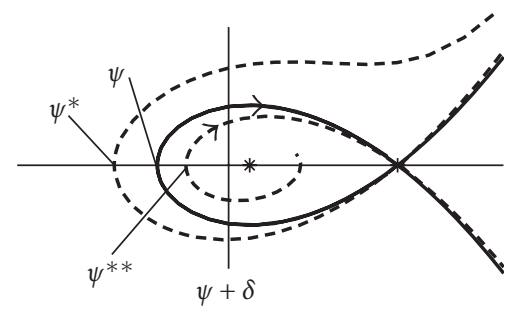

Figure 3.4. Separatrices related to the saddle point $(1,0)$ for $a=0$ (solid lines) and $a>0$ (dashed lines).

To determine (in the linear approximation) the slopes of the tangent lines to separatrices passing through the saddle $(1,0)$, we compute corresponding eigenvalues of the Jacobian matrix evaluated at this steady state from the equation

$$
\operatorname{det}\left(\begin{array}{cc}
-\lambda & 1 \\
2(1-\phi) & a-\lambda
\end{array}\right) \equiv \lambda^{2}-a \lambda-2(1-\phi)=0 .
$$

Thus,

$$
\lambda_{1,2}=\frac{a}{2} \pm \sqrt{\frac{a^{2}}{4}+2(1-\phi)}
$$

and $\lambda_{1}=a / 2-\sqrt{a^{2} / 4+2(1-\phi)}<0$, while $\lambda_{2}=a / 2+\sqrt{a^{2} / 4+2(1-\phi)}>0$.

The tangent line to the separatrix entering this saddle point for $\eta_{0} \rightarrow+\infty$ is defined by equation $p=\lambda_{1}(\tilde{u}-1)$, and the tangent line to the separatrix entering saddle for $\eta_{0} \rightarrow$ $-\infty$ (or, equivalently, for $\eta_{1} \rightarrow+\infty$ ) has equation $p=\lambda_{2}(\tilde{u}-1)$.

Let us impose an additional condition, that is, assume that $a$ is sufficiently small, to obtain a phase portrait for the case $a \neq 0$ from the phase portrait for $a=0$ using asymptotics in $a$.

First, we note that, compared to the case $a=0$, for increasing $a>0$ the negative $\lambda_{1}$ decays in absolute value, and positive $\lambda_{2}$ grows. In Figure 3.4 corresponding separatrices for $a=0$ and $a>0$ are shown (the changes in the slopes of the tangent lines to separatrices at $(0,1)$ are evident from the figure).

Let us follow the separatrix with the tangent slope $\lambda_{2}$ for growing $\eta_{0}$ (or, equivalently, for decaying $\eta_{1}$ ). Let us look for the solution of (3.18) in the form of an expansion

$$
p=p_{0}+a p_{1}
$$

Substituting the above into (3.18), in the leading order approximation we obtain

$$
p_{0} \frac{d p_{0}}{d \tilde{u}}=\left(\tilde{u}^{2}-1\right)(\tilde{u}-\phi) .
$$


This separatrix (corresponding to $a=0$ ) is the lower part of the closed loop $\left(p_{0}<0\right)$ shown in Figure 3.4. In the next order approximation, we write

$$
p_{0} \frac{d p_{1}}{d \tilde{u}}+p_{1} \frac{d p_{0}}{d \tilde{u}}=\frac{d}{d \tilde{u}}\left(p_{0} p_{1}\right)=p_{0}
$$

Thus,

$$
\begin{aligned}
p_{0} p_{1} & =\int_{1}^{\tilde{u}} p_{0}(\eta) d \eta, \\
p_{1} & =\frac{1}{p_{0}} \int_{1}^{\tilde{u}} p_{0}(\eta) d \eta<0 .
\end{aligned}
$$

This expression will be used for the values of $\tilde{u}$ changing between 1 and $\psi+\delta$ where $p_{0}<0$. (From the expression for $p_{1}$, and the fact that $p_{0}<0$, it follows immediately that $p_{1}<0$ as well.) At $\tilde{u}=\psi+\delta$ the roles of $\tilde{u}$ and $p$ must be changed. We now must consider the equation

$$
\frac{d \tilde{u}}{d p}\left(a p+\left(\tilde{u}^{2}-1\right)(\tilde{u}-\phi)\right)=p
$$

with condition

$$
\tilde{u}\left(p^{*}\right)=\tilde{u}\left(p_{0}(\psi+\delta)+a p_{1}(\psi+\delta)\right)=\psi+\delta .
$$

We construct its solution in the form of an expansion

$$
\tilde{u}=u_{0}+a u_{1} .
$$

We now have for the leading order (after substitution of the above expansion into the equation and condition):

$$
\frac{d u_{0}}{d p}\left(\left(u_{0}^{2}-1\right)\left(u_{0}-\phi\right)\right)=\frac{d u_{0}}{d p} F\left(u_{0}\right)=p
$$

where $F(u)=\left(u^{2}-1\right)(u-\phi)$. Thus,

$$
\begin{gathered}
\frac{d u_{0}}{d p}=\frac{p}{F\left(u_{0}\right)}, \\
u_{0}\left(p_{0}(\psi+\delta)\right)=\psi+\delta,
\end{gathered}
$$

which describes the same closed loop (homoclinic orbit) corresponding to $a=0$.

Next,

$$
\frac{d u_{1}}{d p} F\left(u_{0}\right)+\frac{d u_{0}}{d p}\left(p+F^{\prime}\left(u_{0}\right) u_{1}\right)=0 .
$$

This equation must be considered with condition

$$
u_{1}\left(p_{0}(\psi+\delta)\right)=-\frac{d u_{0}}{d p}\left(p_{0}(\psi+\delta)\right) \times p_{1}(\psi+\delta) .
$$


Since on the homoclinic orbit in the vicinity of $\tilde{u}=\psi$ and for $p<0$ we have $d p_{0} / d \tilde{u}<0$, then $d u_{0} / d p<0$. Taking into account that for $p_{0}<0$ it follows from (3.26) that $p_{1}<0$, we finally get

$$
u_{1}\left(p_{0}(\psi+\delta)\right)<0
$$

From the equation for $u_{1}$, we obtain

$$
\frac{d u_{1}}{d p} F\left(u_{0}\right)+\frac{p}{F\left(u_{0}\right)}\left(p+F^{\prime}\left(u_{0}\right) u_{1}\right)=0
$$

or

$$
\begin{aligned}
\frac{d u_{1}}{d p} & =-\frac{p}{F^{2}\left(u_{0}\right)}\left(p+F^{\prime}\left(u_{0}\right) u_{1}\right) \\
& =-u_{1} \frac{p F^{\prime}\left(u_{0}\right)}{F^{2}\left(u_{0}\right)}-\frac{p^{2}}{F^{2}\left(u_{0}\right)}=-u_{1}\left(\frac{d u_{0}}{d p}\right) \frac{F^{\prime}\left(u_{0}\right)}{F\left(u_{0}\right)}-\frac{p^{2}}{F^{2}\left(u_{0}\right)}
\end{aligned}
$$

The solution of this equation can be written as follows (here we use notation $p_{0}^{*}=p_{0}(\psi+$ $\delta))$ :

$$
\begin{aligned}
u_{1}(p)= & u_{1}\left(p_{0}^{*}\right) \exp \left(-\int_{p_{0}^{*}}^{p} \frac{F^{\prime}\left(u_{0}\right)}{F\left(u_{0}\right)} \frac{d u_{0}}{d \eta} d \eta\right) \\
& -\int_{p_{0}^{*}}^{p} \frac{\xi^{2}}{F^{2}\left(u_{0}\right)} \exp \left(-\int_{\xi}^{p} \frac{F^{\prime}\left(u_{0}\right)}{F\left(u_{0}\right)} \frac{d u_{0}}{d \eta} d \eta\right) d \xi \\
= & u_{1}\left(p_{0}^{*}\right) \exp \left[\left.(-\ln F)\right|_{p_{0}^{*}} ^{p}\right]-\int_{p_{0}^{*}}^{p} \frac{\xi^{2}}{F^{2}\left(u_{0}\right)} \exp \left[\left.(-\ln F)\right|_{\xi} ^{p}\right] d \xi \\
= & u_{1}\left(p_{0}^{*}\right) \frac{F\left(u_{0}\left(p_{0}^{*}\right)\right)}{F\left(u_{0}(p)\right)}-\frac{1}{F\left(u_{0}(p)\right)} \int_{p_{0}^{*}}^{p} \frac{d u_{0}}{d \xi} \xi d \xi .
\end{aligned}
$$

Taking into account that $d u_{0} / d p$ is of order $p$ for small $p$, we may omit the second term in the above expression. Then, approximately,

$$
u_{1}(p)=u_{1}\left(p_{0}^{*}\right) \frac{F\left(u_{0}\left(p_{0}^{*}\right)\right)}{F\left(u_{0}(p)\right)}
$$

and thus, $u_{1}(p)$ has the same sign as $u_{1}\left(p_{0}^{*}\right)$ :

$$
u_{1}(p)<0
$$


The value $\psi^{*}$ (abscissa of a point corresponding to $p=0$ ) is defined in the vicinity of the left-most point of the separatrix loop by the formula

$$
\psi^{*}=\psi+a u_{1}(0)=\psi+a u_{1}\left(p_{0}^{*}\right) \frac{F\left(u_{0}\left(p_{0}^{*}\right)\right)}{F(\psi)}<\psi
$$

(The inequality in the above formula follows from (3.40), relation $u_{0}(0)=\psi$, and the fact that $F(\psi)$ and $F\left(u_{0}\left(p_{0}^{*}\right)\right)$ have the same sign.)

When $p$ passes through zero, the trajectory moves into the upper half of the phase plane. The construction of an approximation for the trajectory may be continued by switching at some point back to $\tilde{u}$ being an independent and $p$ being a dependent variable.

In a similar manner we can construct the trajectory corresponding to $\lambda_{1}<0$ and intersecting the $\tilde{u}$-axis $(p=0)$ at a point with abcissa $\psi^{* *}>\psi$ (see Figure 3.4).

Thus, the phase portrait of (3.16) has changed compared to the phase portrait of (3.2). As a result of that the changes will also be observed in the motion of the fronts leading to switching between the upper and the lower boundary layer type solutions shown in Figure 3.2. In particular, for $a=0$ the destruction of the boundary layer transitions at the left and the right boundaries of the spatial interval occurs via the two moving fronts that appear simultaneously for the value of $\phi=3 / 8$. On the other hand, for $a>0$ the moving fronts will always start at the left boundary of the interval $[0,1]$ for some value of $\phi=\phi_{r}<3 / 8$ (for transitions from the upper to the lower boundary layer type solution), and for a value $\phi=\phi_{l}>-3 / 8$ (for transitions from the lower to the upper boundary layer type solution). This happens because for $a>0$ as $\phi$ increases above $\phi_{r}$ (decreases below $\phi_{l}$ ) the vertical line $\tilde{u}=0$ ceases to intersect the separatrix going to saddle point $(1,0)$ (or separatrix tending to $(-1,0))$ as $\eta_{0} \rightarrow \infty$ and describing the left transition layer, while it still intersects the separatrix going to $(1,0)$ (or separatrix tending to $(-1,0)$ ) as $\eta_{1} \rightarrow \infty$ and describing the right transition layer.

A more detailed illustration of the behavior of the boundary layer transitions at both sides of the spatial interval $[0,1]$ is given in Figures 3.5 and 3.6. Explanations of the relation between changing values of $\phi$ and switching from the upper to the lower boundary layer type solution and back are also presented below.

In Figure 3.5 we show schematic representations of phase portraits for the boundary layer transitions corresponding to various stages of time evolution of the periodic solution that exists for $a>0$. Same as in Figure 3.3, the bold solid line corresponds to the left boundary layer transition (near $x=0$ ), and the dashed line corresponds to the right boundary layer transition (near $x=1$ ). Also, to simplify notation in Figure 3.5, tilde is omitted, that is, $u$ is used instead of $\tilde{u}$. Arrows show the directions of increase in $\eta_{0}$ (solid line) and in $\eta_{1}$ (dashed line). In Figure 3.6 characteristic instantaneous profiles of the solution are shown in coordinates $(x, u)$ for certain values of $t$.

The following values of $\phi(t)$ produce various phase portraits shown in Figure 3.5 (and characteristic instantaneous solution shapes shown in Figure 3.6):

(a) $\phi(t)<\phi_{r}<3 / 8$-increase of $\phi(t)$; characteristic solution shape for this case is presented in Figure 3.6(a); 


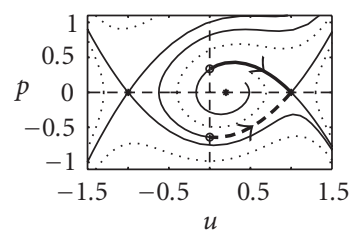

(a)

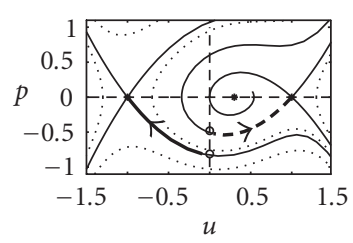

(c)

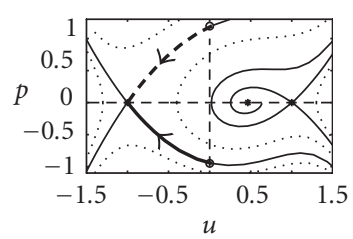

(e)

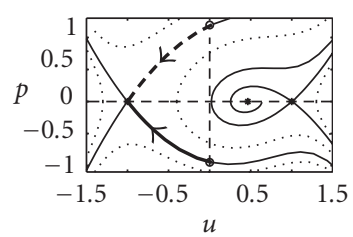

(g)

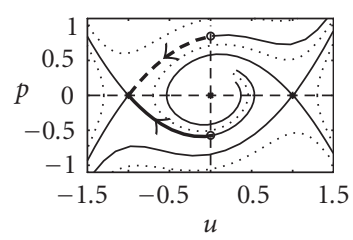

(i)

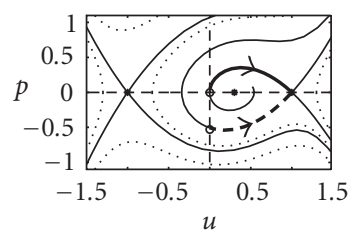

(b)

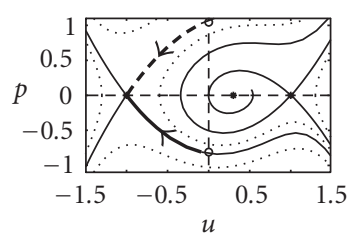

(d)

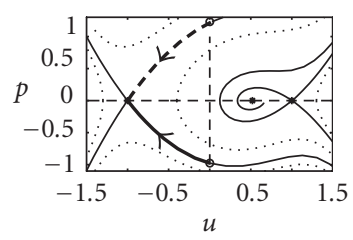

(f)

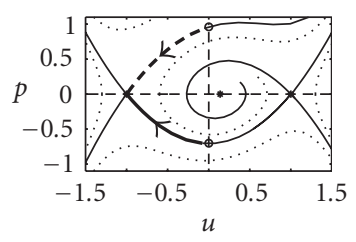

(h)

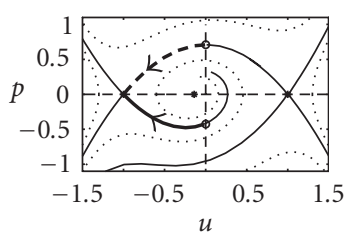

(j)

Figure 3.5. Phase portraits illustrating the time dynamics of the boundary layer transitions present near the left (solid line) and the right (dashed line) boundaries of the interval $x \in[0,1]$ for $a>0$. 


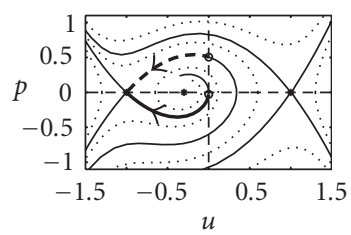

(k)

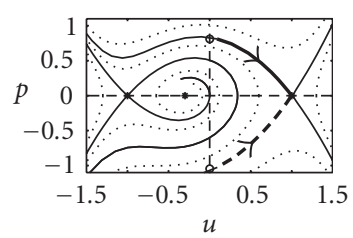

(m)

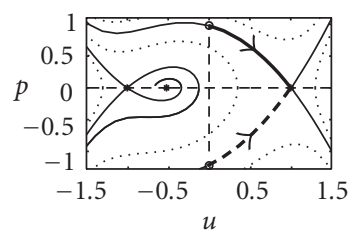

(o)

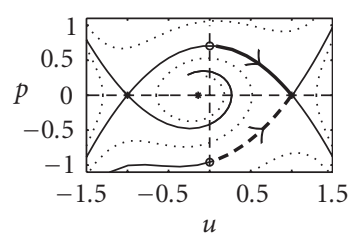

(q)

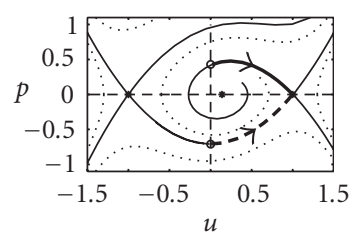

(s)

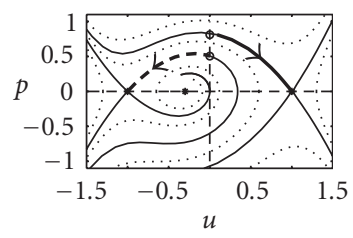

(l)

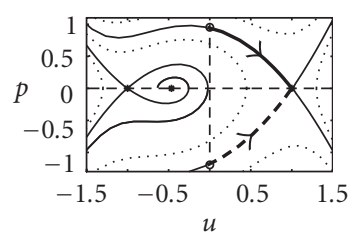

(n)

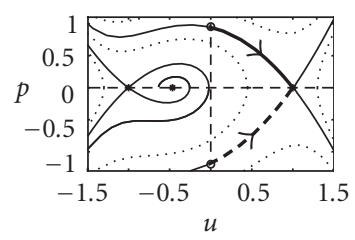

(p)

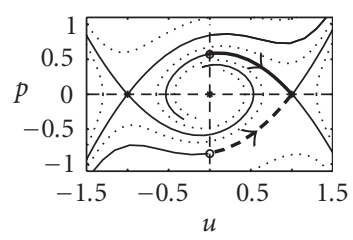

(r)

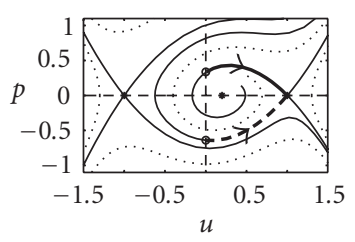

(t)

Figure 3.5. (continued). 
(b) $\phi(t)=\phi_{r}<3 / 8$-maximal critical value; characteristic solution shape is shown in Figure 3.6(b);

(c) $\phi(t)=\phi_{r}+0$ - the start of a non-symmetric run; switching from upper to lower boundary layer type solution via a layer moving from left to right; characteristic solution behavior is illustrated in Figure 3.6(c);

(d) $\phi(t)=\phi_{r}+0$ - the non-symmetric run phase is completed; characteristic lower boundary layer type solution is shown in Figure 3.6(d);

(e), (f) $\phi_{r}<\phi(t)<\phi_{\max }$-increase of $\phi(t)$; characteristic solution shape is in Figure 3.6(d);

(g), (h), (i), (j) $-3 / 8<\phi_{l}<\phi(t)<\phi_{\max }$-decrease of $\phi(t)$; the same characteristic solution shape shown in Figure 3.6(d);

(k) $\phi(t)=\phi_{l}>-3 / 8-$ minimal critical value; characteristic solution shape is shown in Figure 3.6(e);

(l) $\phi(t)=\phi_{l}-0$ —-the start of another non-symmetric run; switching from lower to upper boundary layer type solution via a threshold moving from the left to the right boundary of the interval $[0,1]$; characteristic solution behavior is illustrated in Figure 3.6(f);

(m) $\phi(t)=\phi_{l}-0$ — the non-symmetric run phase is completed; characteristic upper boundary layer type solution is shown in Figure 3.6(a);

(n), (o) $\phi_{\min }<\phi(t)<\phi_{l}$ —decrease of $\phi(t)$; characteristic solution shape is in Figure 3.6(a);

(p), (q), (r), (s), (t) $\phi_{\min }<\phi(t)<\phi_{r}$-increase of $\phi(t)$; same characteristic solution shape shown in Figure 3.6(a).

Stage (a) follows stage $(t)$, which completes the cycle.

Once again, we emphasize an important new feature that appears for $a>0$ compared to the case of $a=0$ : the moving layers that lead to switching between the lower and the upper boundary layer type solutions now always start on the left boundary of the spatial interval $[0,1]$.

The description of a moving front corresponding to transition, for example, from the upper to the lower boundary layer type solution (shown in Figure 3.6(c)) is similar to that presented for the case $a=0$. We may use the same formula (3.8) with $r_{0}$ now defined from the equation

$$
\varepsilon \frac{d r_{0}}{d t}=\sqrt{2}\left(\phi(t)-\frac{a}{\sqrt{2}}\right) .
$$

Then the following conjecture holds.

Conjecture expression (3.8) in which $r_{0}$ is defined by (3.42) satisfies (3.15) with discrepancy of order $O(\varepsilon)$.

Similar phenomenon is also observed for $a<0$. However, in this case the perturbations of the homoclinic loop in the $(\tilde{u}, p)$ plane will lead to corrections for $\psi$ of opposite signs compared to those computed for the case of $a>0$. Corresponding separatrices together with $\psi^{*}$ and $\psi^{* *}$ are presented in Figure 3.7. Analysis, similar to that performed for $a>0$ case, shows that for $a<0$ the moving thresholds leading to switches between the upper 


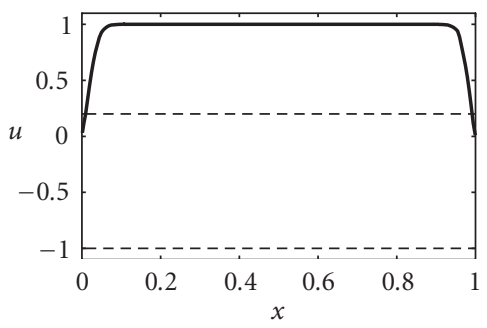

(a)

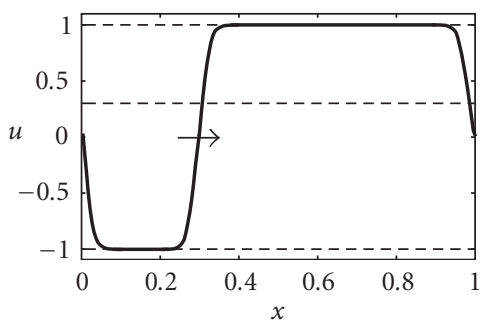

(c)

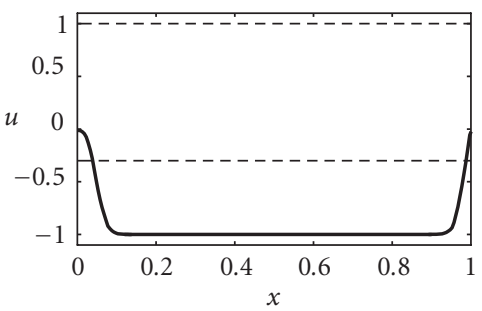

(e)

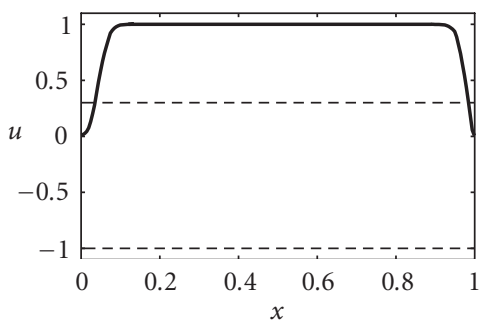

(b)

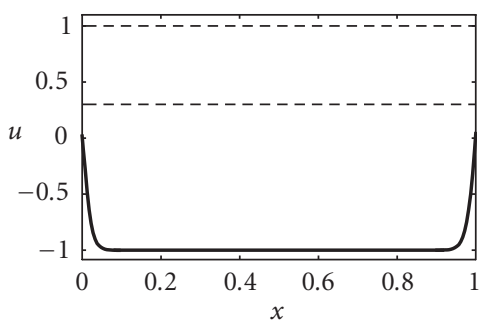

(d)

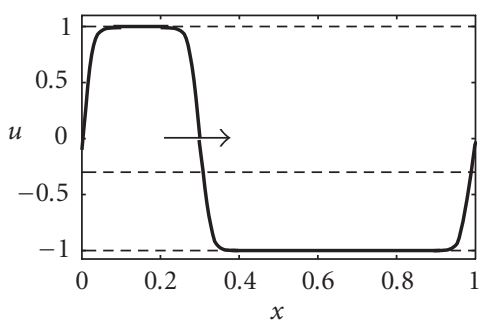

(f)

Figure 3.6. Sample phases of the periodic solution behavior illustrating the switching between the upper and the lower boundary layer type solutions. Now the transition always starts at the left end point of the spatial interval $x \in[0,1]$.

and the lower boundary layer type solutions will now be formed at the right boundary and move towards the left boundary of the spatial interval $[0,1]$.

\section{Conclusions}

In this paper we presented the analysis of alternating boundary layer type solutions for singularly perturbed parabolic equations. Compared to the cases considered earlier, we addressed the situations where the non-linearities do not depend on spatial variable explicitly. The problems with and without convection-like terms (i.e., the terms depending 


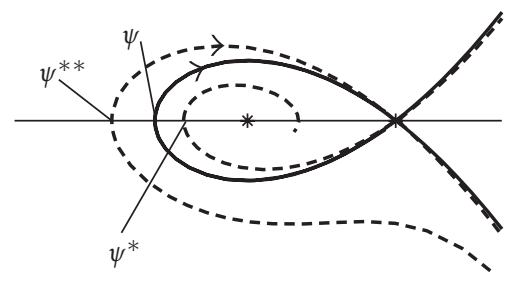

Figure 3.7. Separatrices related to the saddle point $(1,0)$ for $a=0$ (solid lines) and $a<0$ (dashed lines).

on the first derivative of the unknown function with respect to spatial variable) were discussed. In the former case, when the explicit dependence on $u_{x}$ is present, the switch from upper to lower boundary layer type solution (and back) is always initiated on one side (left or right boundary) of the one-dimensional spatial domain. The actual side of initiation is defined by the sign of the convection-like term. In the latter case, without explicit dependence on $u_{x}$, the transition starts symmetrically on both sides of the onedimensional spatial domain.

Here, for problem with $a \neq 0$, we only discussed the case of fast transitions between the two longer lasting stages of the periodic solution where the moving layer runs from one side of the spatial domain to the other without interruptions. Under certain conditions (e.g., on the magnitude of $a$, etc.) the following more complex scenario is possible: the moving layer starts at one (e.g., left) boundary of the domain, and after a short time interval, before the first layer reaches the right boundary, another moving layer is initiated at the right boundary. The two layers will move non-symmetrically and meet (and destroy) each other at some point in the interior of the spatial domain of interest (not in the middle of the domain!). This problem will be addressed in the future and corresponding analysis will be published elsewhere.

Finally, we would like to briefly comment on conclusions following from the analysis and related to sensitivity and possibilities for tuning of bio-switches that are modeled by the singularly perturbed parabolic equations studied above. Heuristically, it could have been expected that the transitions must occur when the value of periodic function $\phi(t)$ equals zero. We note, however, that the actual transitions between the steady states in bistable problems I and II occurred when the slowly changing function $\phi(t)$ passed beyond some critical values (e.g., $3 / 8$ and $-3 / 8$ for bistable problem I). In fact, the delay in starting the transitions, that is, how much $\phi(t)$ had to change compared to 0 for the switching wave to form, was defined not only by the structure of the right-hand sides of the equations, but also by the choice of zero Dirichlet boundary conditions. The critical values mentioned above will change if the Dirichlet boundary conditions are chosen to be non-symmetric: for example, if $u(0, t, \varepsilon)<0<u(1, t, \varepsilon)$ in bistable problem $\mathrm{I}$, then the transitions for increasing $\phi(t)$ will always start at the left boundary $x=0$, and will occur for $\phi_{r}<3 / 8$, while for decreasing $\phi(t)$ they will always start at the right boundary $x=1$, and will occur for $\phi_{l}>-3 / 8$. This means that the sensitivity of the bio-switches, that is, how much $\phi(t)$ must grow or decay compared to zero before the transition starts, could 
be changed by appropriate choice of the boundary conditions. One may also tune the parameters of the above models to produce a specific required delay. It also follows from the analysis that the bio-switch models with convection-like terms are more sensitive compared to similar models without convection.

\section{Acknowledgments}

The authors would like to thank Professor Nicholas D. Alikakos for carefully reading the manuscript and for making numerous helpful suggestions. This work was supported in part by the Russian Foundation for Basic Research, Project no. 03-01-04001.

\section{References}

[1] N. D. Alikakos, P. W. Bates, and X. Chen, Periodic traveling waves and locating oscillating patterns in multidimensional domains, Transactions of the American Mathematical Society 351 (1999), no. 7, 2777-2805.

[2] J. Keener and J. Sneyd, Mathematical Physiology, Interdisciplinary Applied Mathematics, vol. 8, Springer, New York, 1998.

[3] J. D. Murray, Mathematical Biology, 2nd ed., Biomathematics, vol. 19, Springer, New York, 1993.

[4] N. N. Nefedov, An asymptotic method of differential inequalities for the investigation of periodic contrast structures: existence, asymptotics, and stability, Differential Equations 36 (2000), no. 2, 298-305.

[5] A. Okubo and S. A. Levin, Diffusion and Ecological Problems: Modern Perspectives, 2nd ed., vol. 14, Springer, New York, 2001.

[6] A. B. Vasil'eva, Periodic solutions to a parabolic problem with a small parameter multiplying the derivatives, Computational Mathematics and Mathematical Physics 43 (2003), no. 7, 932-943.

[7] A. B. Vasil'eva, V. F. Butuzov, and L. V. Kalachev, The Boundary Function Method for Singular Perturbation Problems, SIAM Studies in Applied Mathematics, vol. 14, SIAM, Pennsylvania, 1995.

[8] A. B. Vasil'eva, A. P. Petrov, and A. A. Plotnikov, On the theory of alternating contrast structures, Computational Mathematics and Mathematical Physics 38 (1998), no. 9, 1471-1480.

Adelaida B. Vasil'eva: Department of Physics, Moscow State University, Moscow 119899, Russia E-mail address: abvas@mathabv.phys.msu.su

Leonid V. Kalachev: Department of Mathematical Sciences, University of Montana, Missoula, MT 59812, USA

E-mail address: kalachev@mso.umt.edu 


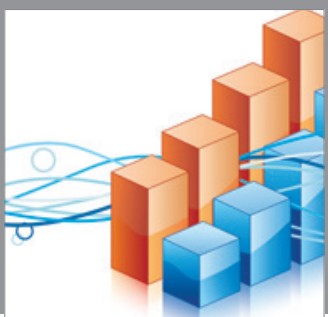

Advances in

Operations Research

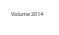

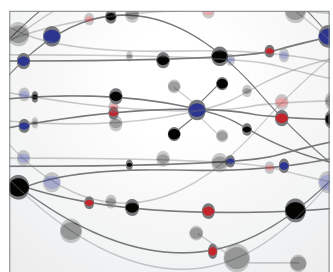

\section{The Scientific} World Journal
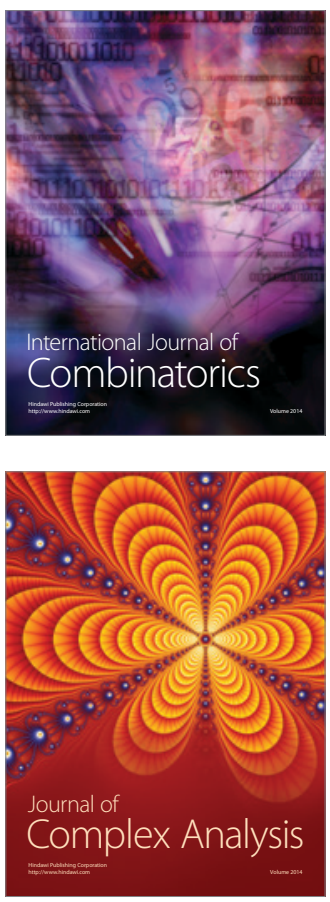

International Journal of

Mathematics and

Mathematical

Sciences
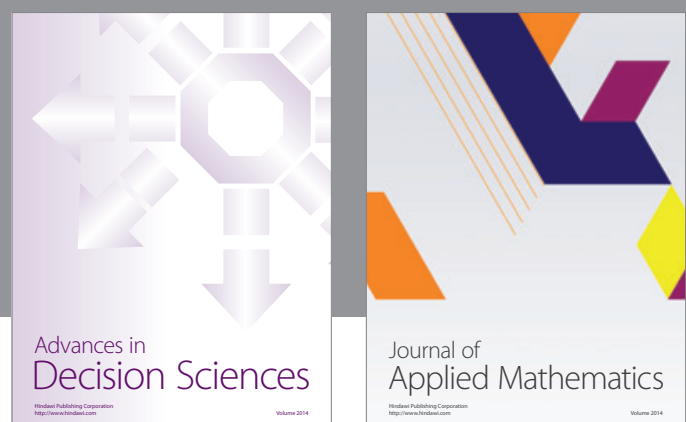

Journal of

Applied Mathematics
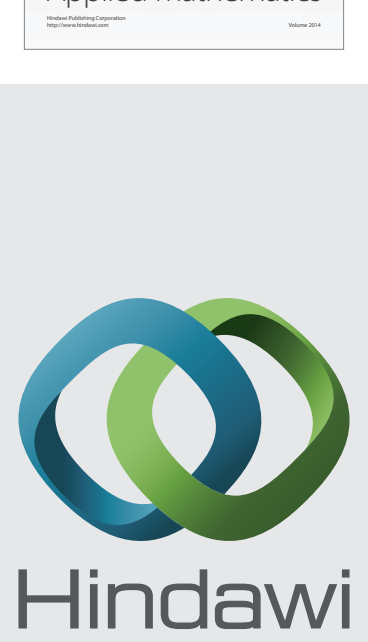

Submit your manuscripts at http://www.hindawi.com
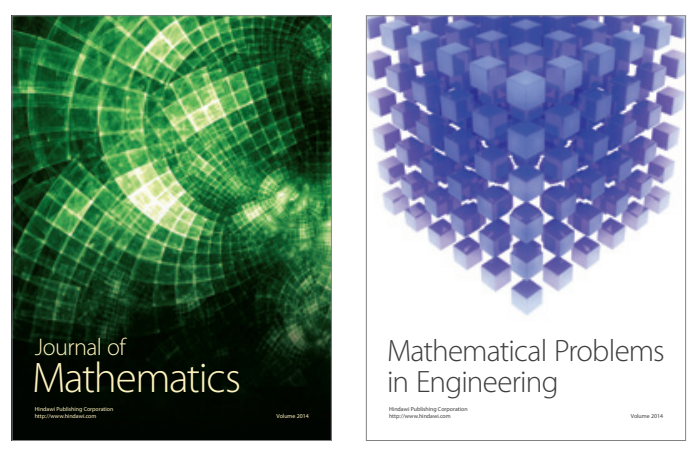

Mathematical Problems in Engineering
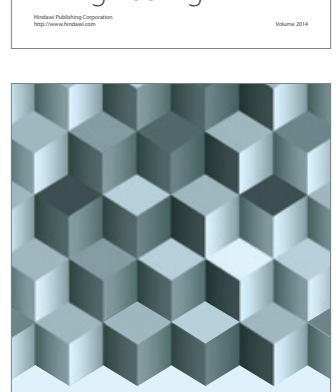

Journal of

Function Spaces
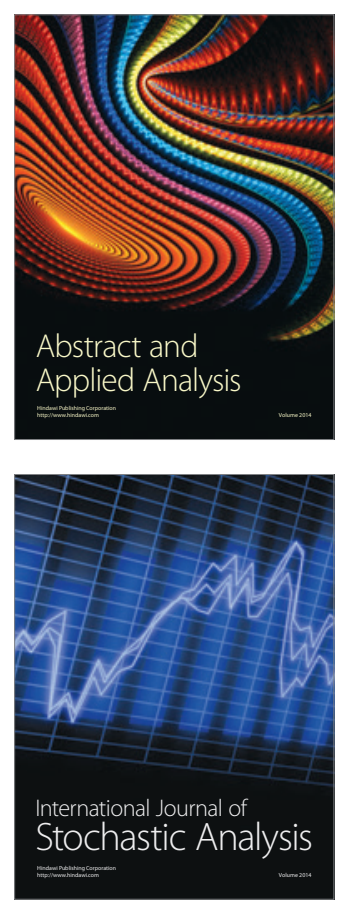

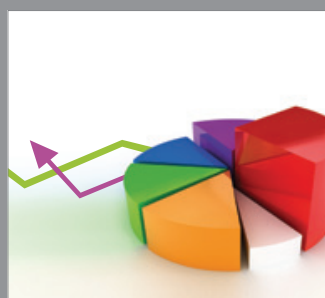

ournal of

Probability and Statistics

Promensencen
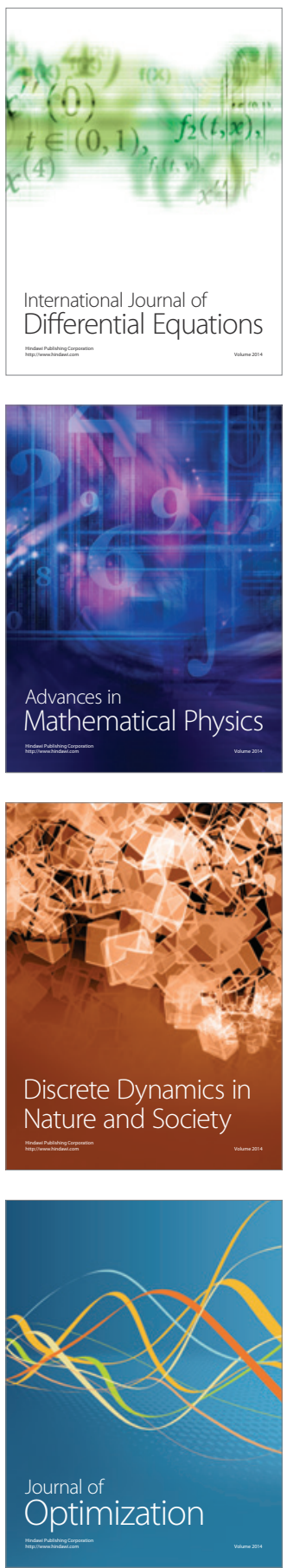